\title{
ÍNDICES DE DESENVOLVIMENTO SUSTENTÁVEL PARA LOCALIDADES: UMA PROPOSTA METODOLÓGICA DE CONSTRUÇÃO E ANÁLISE
}

\author{
Maria de Fátima Martins \\ Doutora em Recursos Naturais da Universidade Federal de Campina Grande - UFCG \\ Professora da Unidade Acadêmica de Administração e Contabilidade da Universidade Federal de \\ Campina Grande - UFCG \\ fatimamartins2005@gmail.com

\section{Gesinaldo Ataíde Cândido} \\ Doutor em Engenharia de Produção pela Universidade Federal de Santa Catarina - UFSC \\ Professor da Universidade Federal de Campina Grande - UFCG \\ gacandido@uol.com.br
}

\section{RESUMO}

O objetivo deste artigo é propor uma metodologia para construção e análise do Índice de Desenvolvimento Sustentável para Municípios (IDSM), a partir da coleta, tratamento e análise de indicadores de sustentabilidade. A elaboração dessa proposta metodológica teve como base o modelo desenvolvido por Sepúlveda (2005) junto ao Instituto Interamericano de Cooperação para Agricultura (IICA) e aplicado no Brasil por Waquil, Shneider, Fileppi, Conterato e Specht (2006) em territórios rurais e o IDS-Brasil 2004, desenvolvido pelo Instituto Brasileiro de Geografia e Estatística (IBGE). A classificação dos níveis de sustentabilidade foi possível a partir da definição de uma escala que indica a sustentabilidade ideal, aceitável, alerta e crítica, cuja representação foi ilustrada por meio de mapas. A aplicação da metolodogia IDSM teve como objeto de estudo os Estados brasileiros e os municípios do Estado da Paraíba. A relevância desse artigo consiste em ampliar o debate sobre o desenvolvimento sustentável, possibilitando uma avaliação das desigualdades regionais e locais, instigando a necessidade de direcionar políticas específicas que levem a um processo de desenvolvimento em bases sustentáveis. Os resultados permitiram disponibilizar um conjunto de informações que servirão de subsídios para a formulação e implementação de políticas públicas que propiciem as condições adequadas para o processo de desenvolvimento local.

Palavras-chave: Desenvolvimento; Indicadores; Municípios; Políticas públicas; Sustentabilidade.

\section{INDEXES FOR SUSTAINABLE DEVELOPMENT LOCALS: A PROPOSAL METHODOLOGY OF CONSTRUCTION AND ANALYSIS}

\begin{abstract}
The objective of this article is to propose a methodology for construction and analysis of the Index of Sustainable Development for Cities (IDSM), from the collection, processing, and analysis of sustainability indicators and classification of specific levels of sustainability of these locals. The elaboration of such a methodological proposal was based on the model developed by Sepúlveda (2005) with the Inter-American Institute for Cooperation on Agriculture (IICA) and implemented in Brazil by Waquil, Shneider, Fileppi, Conterato and Specht (2006) in rural areas and IDS-Brasil 2004 developed by the Brazilian Institute of Geography and Statistics (IBGE). The classification levels of sustainability were possible from the definition of a scale that indicates sustainability as ideal, acceptable, warning, and critical, and whose representation was illustrated by on maps. The implementation of metolodogia IDSM had as its object of study communes of the state of Paraiba. The relevance of this article is to broaden the debate on sustainable development, enabling an assessment of local and regional inequalities. This would ultimately prompt the need to target specific troubled areas and lead to a process of comprehensive development, and on a sustainable footing. The results can provide a set of information that serve as subsidies for the formulation and implementation of public policies that provide the right conditions for the local development process.
\end{abstract}

Keywords: Development; Indicators; Cities; Publics Policies; Sustainability. 


\section{INTRODUÇÃO}

O modelo de desenvolvimento adotado nos últimos tempos, baseado no crescimento das relações de produção e consumo, tem como principais implicações o crescimento do nível de degradação dos recursos naturais, da poluição ambiental e nos níveis de desigualdade social e de concentração de riqueza. Como decorrência dessa situação, surge o conceito de desenvolvimento sustentável e da sustentabilidade, que procuram reduzir tais implicações, a partir do entendimento das fragilidades do modelo vigente e da emergência da necessidade de uma nova concepção de desenvolvimento de forma equilibrada e equitativa.

Esta nova abordagem do desenvolvimento resgata a necessidade da incorporação de um conjunto de dimensões e indicadores que procuram compreender, de forma sistêmica, o processo de construção do desenvolvimento, incorporando os aspectos sociais, econômicos, político, institucionais, ambientais, demográficos, culturais, etc. Os seus conceitos e aplicações vêm sendo sistematicamente discutidos pelos movimentos sociais, instituições de ensino e pesquisa, ONGs, políticas governamentais, estratégias empresariais, entre outras iniciativas com algum tipo de vínculo com políticas e ações para geração do desenvolvimento.

No cerne dessas questões que envolvem o desenvolvimento sustentável e a sustentabilidade, uma questão primordial são as múltiplas formas de percepção acerca do tema, uma vez que é permeado de valores baseado em diversos interesses que os vários atores sociais e institucionais possam ter sobre seus conceitos e práticas. Em razão de suas contradições e paradoxos, o entendimento do seu real significado dificulta a obtenção de melhores resultados em sua aplicação.

No entanto, para o National Research Council (1999), as maiores divergências atuais em torno da problemática do desenvolvimento sustentado consistem em saber o que deve ser sustentado; o que deve ser desenvolvido; os tipos de relação que prevalecem entre o sustentado e o desenvolvido; e a extensão do futuro a ser considerado; além de assegurar e manter o crescimento econômico e o desenvolvimento respeitando os limites estabelecidos pela natureza.

Lafer (1996) destaca que o termo desenvolvimento sustentável é claramente carregado de valores, nos quais existe uma forte relação entre os princípios, a ética, as crenças e os valores que fundamentam uma sociedade ou comunidade e sua concepção de sustentabilidade. A diferença nas definições decorre das diferentes abordagens que se têm sobre o conceito, sendo assim, o grau de sustentabilidade é relativo, dependendo do ponto de vista considerado, isto é, em razão do campo ideológico ambiental ou dimensão em que cada ator se coloca.

De acordo com Ruthes e Nascimento (2006), o desenvolvimento deve ser pensado sistematicamente, de forma coletiva e participativa, gerando efeitos significativos na promoção da sustentabilidade.

Dada a complexidade que envolve o desenvolvimento sustentável é necessário encontrar indicadores que captem todos os aspectos relevantes para o processo de desenvolvimento em bases sustentáveis e descrevam, de forma compreensiva, uma realidade mutável, dinâmica e diversa, além de evidenciar aspectos que revelem tendências ou perspectivas futuras. Neste sentido, os aspectos relacionados ao desenvolvimento sustentável são complexos e requerem formas diversas de análises, a partir do número adequado de indicadores e variáveis que sejam os mais consistentes e fidedignos para retratar um dado contexto. Surge daí, a necessidade da criação ou adaptação de indicadores de sustentabilidade que permitam contribuir para a superação dos desafios decorrentes da viabilização do desenvolvimento sustentável, tendo como base metodologias que permitam retratar a realidade e forneçam subsídios e informações que favoreçam as interações entre os sistemas humanos e ambientais orientados para resultados sustentáveis.

De acordo com Schmidt, Cazella e Turnes (1998), a avaliação das ações de desenvolvimento constitui um requisito essencial para a obtenção da sustentabilidade em um determinado território, constituindo-se em um elemento-chave para a formulação de políticas e a tomada de decisões. Em razão disso, tem surgido uma série de iniciativas que propõem a adoção de indicadores de 
sustentabilidade, nas diversas áreas relacionadas ao desenvolvimento das sociedades.

Este estudo parte da percepção de que a avaliação do desenvolvimento sustentável deve considerar as características e diversidades locais, como forma de melhor retratar esse processo e oferecer subsídios para a elaboração e implementação de políticas públicas de desenvolvimento local.

A premissa central deste estudo é de que o desenvolvimento deve ser encarado como uma construção coletiva, visando à consolidação de uma nova realidade local. Sendo assim, monitorar e avaliar o andamento deste processo é fundamental para garantir a sustentabilidade. Esta avaliação deve considerar as próprias dimensões de um desenvolvimento efetivo que se diferencie, portanto, do crescimento econômico, e leve em consideração a necessidade de ser socialmente justo, economicamente viável e ambientalmente responsável. Como esse processo é caracterizado pela contínua mudança, é preciso possuir instrumentos de avaliação simples e que possam captar periodicamente os resultados positivos e negativos das ações implementadas. Estes instrumentos devem servir como antenas, captando as necessidades de alterações de rumo, identificando potencialidades e vulnerabilidades, bem como, as diversidades regionais e locais existentes.

Nessa perspectiva, o objetivo desse artigo consiste em propor uma metodologia para construção e análise do Índice de Desenvolvimento Sustentável para Municípios (IDSM), a partir da coleta, tratamento e análise de indicadores de sustentabilidade. Essa metodologia, tendo como objeto de estudo os estados brasileiros e os municípios da Paraíba, possibilita a disponibilização de um conjunto de informações por meio de índices, que agregados em dimensões propiciam a criação do IDSM e a classificação do nível de sustentabilidade dessas localidades.

A metodologia proposta reconhece a necessidade da abordagem sistêmica para tratar das questões da sustentabilidade, sendo assim, as informações foram organizadas numa perspectiva ampla e integrada envolvendo os aspectos sociais, demográficos, econômicos, político-institucional, ambiental e cultural, no sentido de fornecer subsídios para a formulação e implementação de políticas públicas que propiciem as condições adequadas para a qualidade da vida da população no momento atual e para as futuras gerações.

Este artigo encontra-se dividido em três seções: a primeira, apresenta o referencial teórico a partir da temática: sustentabilidade, as dimensões da sustentabililidade, indicadores e sistemas de indicadores; a segunda, aborda os aspectos metodológicos; a terceira, apresenta os resultados do estudo e, por fim, as considerações finais.

\section{FUNDAMENTAÇÃO TEÓRICA}

\subsection{Sustentabilidade}

A interdisciplinaridade que envolve as temáticas desenvolvimento sustentável e sustentabilidade faz com que esses temas estejam presentes nas discussões nos mais diversos campos da ciência, na iniciativa privada e pública, nas organizações não-governamentais e em toda a sociedade. Diante da relevância e das implicações dessas temáticas, busca-se chegar a um entendimento dos conceitos que permeiam todas essas discussões e, principalmente, a operacionalização desses conceitos para atingir o desenvolvimento em bases sustentáveis.

O termo desenvolvimento sustentável foi reconhecido a partir do Relatório Blundtland ou como ficou conhecido Nosso Futuro Comum(1991) da Comissão Mundial sobre Meio Ambiente e Desenvolvimento, como um processo de transformação no qual a exploração dos recursos, a direção dos investimentos, a orientação do desenvolvimento tecnológico e as mudanças institucionais se harmonizem e reforçam o potencial presente e futuro, para atender as necessidades e aspirações humanas.

A sustentabilidade significa a possibilidade de se obterem continuamente condições iguais ou superiores de vida em dado ecossistema, visando a manutenção do sistema de suporte da vida. Sendo assim, a sustentabilidade relaciona-se com a melhor qualidade da vida das populações, a partir da

Revista de Gestão Social e Ambiental - RGSA, São Paulo, v. 5, n. 3, p. 03-19, set./dez. 2011. 
capacidade de suporte dos ecossistemas. Franco (2000) reforça essa idéia ao destacar que, quando a população extrapola a capacidade de suporte afeta a qualidade de vida, pois a liberdade de escolha de estilo de vida é partida. Essa relação entre a sustentabilidade e a qualidade de vida pode ser definida como o grau de prazer, satisfação e realizações alcançadas por um indivíduo no seu processo de vida.

A complexidade existente em torno das interações entre os sistemas humanos e sistemas ambientais, faz com que o debate sobre o desenvolvimento sustentável seja amplo e multidisciplinar, além de ser carregado de nuances que dificultam sua aplicabilidade e, por conseguinte, o alcance de resultados realmente sustentáveis sob a ótica social, ambiental, demográfica, política, econômica, institucional, cultural e institucional. Diante dessa abordagem, ao buscar alcançar o desenvolvimento sustentável é imprescindível reconhecer que os sistemas estão interligados e exercem influências mútuas, ao mesmo tempo que, cada sistema exige interferências diferenciadas de acordo com o nível de evolução que se encontra, o reconhecimento de suas características e a capacidade de atuação e interação estabelecida com o contexto no qual está inserido. Sendo assim, o desenvolvimento sustentável exige posturas diferenciadas conforme as peculiaridades das interações homem-natureza, que, por sua vez, exigem posturas adequadas às características do ambiente.

Para Ribeiro (2000), a mensuração da sustentabilidade de um processo de desenvolvimento é fundamental para operacionalizar o conceito de desenvolvimento sustentável. O apoio oferecido pela mensuração, possibilita que a decisão política acerca do desenvolvimento seja exequível com os objetivos da sustentabilidade. Sendo assim, a meta básica da mensuração da sustentabilidade do desenvolvimento é fornecer elementos concretos para apoiar a decisão, sendo um poderoso recurso de apoio ao planejamento de ações futuras. A concepção de indicadores de sustentabilidade emerge nesse plano como suportes fundamentais para a atividade de mensurar, possibilitando que as escolhas políticas movam-se em direção à sustentabilidade, pela criação de conexões entre o atual estágio de desenvolvimento e o estado de sustentável no futuro.

Nesse contexto, o IBGE (2004) define indicadores como ferramentas constituídas por uma ou mais variáveis que, associadas por meio de diversas formas, revelam significados mais amplos sobre os fenômenos, sendo assim, os indicadores de desenvolvimento sustentável são instrumentos essenciais para guiar a ação e subsidiar o acompanhamento e a avaliação do progresso alcançado rumo ao desenvolvimento sustentável.

Van Bellen (2005) enfatiza que os indicadores devem ser entendidos como variáveis, ou seja, a representação operacional de um atributo (qualidade, característica, propriedade) de um sistema, cujo objetivo principal consiste em agregar e quantificar informações ressaltando sua significância, visando melhorar o processo de comunicação e o entendimento dos fenômenos complexos.

Em busca de uma forma de mensurar a sustentabilidade, os indicadores são essenciais para concretizar um processo de desenvolvimento em bases sustentáveis, pela operacionalização de um conjunto de variáveis que são relevantes para a comunicação de informações e, por conseguinte, para a compreensão da realidade investigada. Dada a complexidade e a interdisciplinaridade que envolve a sustentabilidade, os indicadores devem ser analisados por meio de sistemas que permitam verificar a interdependência dessas variáveis para gerar resultados confiáveis e que retrate tal realidade.

\subsection{Sistemas de indicadores de sustentabilidade}

Como o conceito de desenvolvimento sustentável abrange uma diversidade de aspectos com enfoques diferenciados, a partir de diferentes dimensões, isso também é refletido nos sistemas de indicadores. Assim sendo, diversos tipos de sistemas têm sido desenvolvidos e utilizados para mensurar a sustentabilidade do desenvolvimento. Van Bellen (2005) identifica alguns desses 
sistemas, sendo eles: Pressure/State/Response (PSR); Driving-force/State/Respone (DSR), adotado pela Comissão de Desenvolvimento Sustentável das Nações Unidas como ferramenta capaz de organizar informações sobre o desenvolvimento; Human Development Index (HDI), desenvolvido pelo Programa das Nações Unidas para o Desenvolvimento; Dashboard of Sustainability (DS), desenvolvido pelo Instituto Internacional para o Desenvolvimento Sustentável denominado painel da sustentabilidade; Barometer of Sustainability (BS), desenvolvido por diversos especialistas ligados ao Instituto World Conservation Union (IUCN) e o Internaciontional Development Research Centre (IDRC); Ecological Footprint Method (EFM), desenvolvido por Wackernagel and Rees; Monitoring environmental progress (MEP), desenvolvido pelo World Bank.

Há uma significativa quantidade de sistemas de indicadores, porém em razão do escopo geográfico ser direcionado para países, surge uma lacuna quanto à disponibilidade de indicadores para municípios, o que prejudica a construção de índices de desenvolvimento específicos para mensurar a sustentabilidade desses espaços geográficos. Para atender aos objetivos deste artigo de criar um índice de sustentabilidade para municípios, optou-se por elaborar um sistema de indicadores, a partir da disponibilidade e acesso dos dados para os municípios brasileiros, tomando como base o IDS Brasil 2004 e os Indices de Desenvolvimento Sustentável para Territórios Rurais, propostos por Waquil, Shneider, Fileppi, Conterato e Specht (2006) tendo como base a abordagem proposta por Sepúlveda (2005).

\subsubsection{O IDS Brasil}

O IDS Brasil foi elaborado pelo Instituto Brasileiro de Geografia e Estatística (IBGE). O trabalho de construção desses indicadores foi inspirado no movimento internacional liderado pela Comissão para o Desenvolvimento Sustentável (CDS), das Nações Unidas. Este movimento, deflagrado a partir de 1992, pôs em marcha um programa de trabalho composto por diversos estudos e intercâmbios de informação, para concretizar as disposições dos capítulos 8 e 40 da Agenda 21 que tratam da relação entre meio ambiente, desenvolvimento sustentável e informações para a tomada de decisões. Em 1996, a CDS publicou o documento Indicators of sustainable development: framework and methodologies, conhecido como "Livro Azul", composto por um conjunto de 134 indicadores, posteriormente reduzidos em uma lista de 57, apresentada no ano de 2000, acompanhada por fichas metodológicas e diretrizes para sua utilização (IBGE, 2004).

Esse sistema de indicadores produzido pelo IBGE tem como referência o Livro Azul e as recomendações adicionais que o sucederam, adaptando seu conteúdo às particularidades brasileiras. A primeira publicação ocorreu em 2002, sendo composta por 50 indicadores. A edição de 2004, que serviu de base para esta proposta contida neste artigo, é constituída por 59 indicadores que, em sua maior parte, correspondem as atualizações da edição de 2002 e a inclusão de 12 novos indicadores como forma de incorporar novas questões à visão que se oferece sobre o desenvolvimento sustentável no País. Depois disso, foram publicadas as versões 2006 e 2008, com poucas modificações em relação às anteriores.

\subsection{2 Índices de desenvolvimento sustentável para territórios rurais}

Esse índice é uma ferramenta metodológica desenvolvida por Sepúlveda (2005) no Instituto Interamericano de Cooperação para a Agricultura (IICA) para verificação de processo de desenvolvimento sustentável em alguns países da América Latina. Essa metodologia consiste na coleta e sistematização de indicadores representativos de um conjunto de dimensões do desenvolvimento sustentável, que permite a realização de avaliações rápidas, bem como, análise comparativa dos níveis de desenvolvimento sustentável em diferentes territórios.

A definição das dimensões e variáveis é orientada pela realidade dos territórios analisados, onde são caracterizados os espaços geográficos de forma multidimensional para uma percepção das diferenças e identidades próprias. 
Considerando que o conjunto de variáveis apresenta diferentes unidades de medidas que impossibilita a agregação em suas respectivas dimensões e a análise adequada da sustentabilidade, é necessário a transformação dessas variáveis em índices para permitir a agregação nas respectivas dimensões. O procedimento adotado ajusta os valores observados das variáveis a escalas cujo valor mínimo é 0 (zero) e valor máximo é igual a 1 (um), criando condições para a agregação em seis dimensões e a estimação do índice de desenvolvimento sustentável. Como essas variáveis apresentam relação positiva ou negativa com o desenvolvimento sustentável, é necessário definir tal relação, cuja operacionalização para o cálculo do índice é feita a partir de fórmulas que reconhecem essas relações e permitem a análise da sustentabilidade por meio da agregação de todos os índices.

A partir desses e outros sistemas de indicadores de sustentabilidade, diversos novos sistemas deverão ser construídos ao longo dos anos em busca de encontrar formas mais consistentes e adequadas para mensurar a sustentabilidade local, regional, nacional e global. Nessa perspectiva, ampliam-se as discussões, a abrangência e caminha-se para a construção de metodologias que gerem resultados mais efetivos e sustentáveis para contextos específicos.

\section{PROCEDIMENTOS METODOLÓGICOS}

A elaboração da metodologia de avaliação dos níveis de sustentabilidade por meio do Índices de Desenvolvimento Sustentável para Municípios (IDSM) teve como objeto de estudo para sua validação, os estados da brasileiros (incluindo o Distrito Federal) e os municípios do Estado da Paraíba. A decisão de estudar todos os estados partiu da necessidade de conhecer a realidade brasileira e as desigualdades regionais, visando organizar um conjunto de informações como subsídio para a elaboração de políticas públicas com foco no desenvolvimento local sustentado. De forma mais específica, partiu-se para um estudo mais detalhado nos municípios do Estado da Paraíba.

Considerando os critérios mundialmente utilizados para a escolha dos indicadores de sustentabilidade, procurou-se em cada um dos indicadores selecionados as seguintes características: a) ser significativo para a realidade investigada e para o enfoque do estudo; b) ser relevante para as decisões que orientam as políticas públicas; c) refletir as mudanças temporais; d) permitir um enfoque integrado e sistêmico; e) utilizar variáveis mensuráveis; f) ser de fácil interpretação e comunicação; e g) ter uma metodologia bem definida, transparente e objetiva aos propósitos da investigação. Além desses critérios, o motivo principal para a escolha dos indicadores foi a disponibilidade de todos os dados para os municípios brasileiros.

Para a definição das dimensões e variáveis, tomou-se como referência a proposta de Waquil, Shneider, Fileppi, Conterato e Specht (2006) no sentido de buscar uma compreensão da sustentabilidade, tendo como base as dimensões social, demográfica, ambiental, econômica, políticoinstitucional e cultural, reunindo um conjunto de variáveis que buscam caracterizar os espaços geográficos de forma multidimensional, por meio da percepção das distinções e identidades próprias. Além disso, a publicação dos indicadores de desenvolvimento sustentável: Brasil 2004(IBGE, 2004), serviu como um guia para a definição das variáveis que permitissem uma avaliação mais completa da sustentabilidade, considerando as peculiaridades e características da realidade dos municípios no contexto local.

A partir dessas referências, foram definidas as seguintes dimensões e suas respectivas variáveis (Quadro 1), cuja descrição, justificativa e a fonte de dados utilizada encontram-se disponíveis em Martins e Cândido (2008): 


\begin{tabular}{|c|c|}
\hline DIMENSÃO & VARIÁVEIS \\
\hline DIMENSÃO SOCIAL & $\begin{array}{l}\text { Esperança de vida ao nascer/ Mortalidade } \\
\text { Infantil/ Prevalência da desnutrição total/ } \\
\text { Imunização contra doenças infecciosas infantis/ } \\
\text { Oferta de Serviços básicos de saúde/ } \\
\text { Escolarização/ Alfabetização/ Escolaridade/ } \\
\text { Analfabetismo funcional/ Famílias atendidas } \\
\text { com programas sociais/ Adequação de moradia } \\
\text { nos domicílios/ Mortalidade por homicídio/ } \\
\text { Mortalidade por acidente de transporte. }\end{array}$ \\
\hline DIMENSÃO DEMOGRÁFICA & $\begin{array}{l}\text { Crescimento da população/ Razão entre a } \\
\text { população urbana e rural/ Densidade } \\
\text { demográfica/ Razão entre a população masculina } \\
\text { e feminina/ Distribuição da população por faixa } \\
\text { etária. }\end{array}$ \\
\hline DIMENSÃO ECONÔMICA & $\begin{array}{l}\text { Produto Interno Bruto per capita/ Participação } \\
\text { da indústria no PIB/ Saldo da balança comercial/ } \\
\text { Renda Familiar per capita em salários mínimos/ } \\
\text { Renda per capita/ Rendimentos provenientes do } \\
\text { trabalho/ Índice de Gini de distribuição do } \\
\text { rendimento. }\end{array}$ \\
\hline DIMENSÃO POLÍTICO-INSTITUCIONAL & $\begin{array}{l}\text { Despesas por função: com assistência social, } \\
\text { educação, cultura, urbanismo, habitação urbana, } \\
\text { gestão ambiental, ciência e tecnologia, desporto } \\
\text { e lazer, saneamento urbano, saúde/ Acesso a } \\
\text { serviço de telefonia fixa/ Participação nas } \\
\text { eleições/ Número de conselhos municipais/ } \\
\text { Número de acessos a justiça/ Transferências } \\
\text { intergovernamentais da União }\end{array}$ \\
\hline DIMENSÃO AMBIENTAL & $\begin{array}{l}\text { Qualidade das águas: aferição de cloro residual, } \\
\text { de turbidez, de coliformes totais/ Tratamento das } \\
\text { águas: tratada em ETAs e por desinfecção/ } \\
\text { Consumo médio per capita de água/ Acesso ao } \\
\text { sistema de abastecimento de água/ Tipo de } \\
\text { esgotamento sanitário por domicílio/ Acesso a } \\
\text { coleta de lixo urbano e rural. }\end{array}$ \\
\hline DIMENSÃO CULTURAL & $\begin{array}{l}\text { Quantidade de: bibliotecas, museus, ginásios de } \\
\text { esportes e estádios, cinemas, Unidades de } \\
\text { Ensino Superior, teatros ou salas de espetáculos, } \\
\text { centros cultural. }\end{array}$ \\
\hline
\end{tabular}

Quadro 1: Dimensão e variáveis da sustentabilidade.

Fonte: MARTINS e CÂNDIDO (2008).

Como as variáveis apresentam diferentes unidades de medida, as mesmas foram transformadas em índices, possibilitando a agregação nas respectivas dimensões para a estimação do Índice de Desenvolvimento Sustentável para Municípios (IDSM). Para isso, tomou-se como base a proposta metodológica desenvolvida pelo Instituto Interamericano de Cooperação para a Agricultura (IICA) para verificação de processo de desenvolvimento sustentável em alguns países da América Latina e utilizada no Brasil por Waquil, Shneider, Fileppi, Conterato e Specht (2006), onde utiliza um procedimento que ajusta os valores das variáveis numa escala com variação cujo valor mínimo é 0 (zero) e o valor máximo é 1 (um). Assim, os índices apresentarão valores com variação entre 0 - 1 .

Considerando que as variáveis que compõem as dimensões apresentam-se como positivas (quanto maior melhor e quanto menor pior) e negativas (quanto menor melhor e quanto maior pior), de acordo com o contexto de suas relações. Neste contexto, a transformação dessas variáveis, que 
possibilitou a agregação adequada desses índices, levou a necessidade de estabelecer o tipo de relação que cada variável apresenta no contexto da sustentabilidade das localidades estudadas. Nessa perspectiva, foram definidos os tipos de relação (positiva ou negativa) que essas variáveis apresentam com o desenvolvimento sustentável, identificados pelo comportamento da variável em que, se aumentar seu valor (indicador), favorece ou desfavorece o processo de desenvolvimento.

A variável apresenta uma relação positiva quando verificado que, quanto maior o indicador melhor será o índice e quanto menor o indicador pior será o índice. A variável apresenta uma relação negativa quando verificado que, quanto maior o indicador pior será o índice; quanto menor o indicador, melhor será o índice.

Após identificar a relação positiva ou negativa da variável com o processo de desenvolvimento sustentável, a operacionalização para o cálculo do índice é feita a partir de fórmulas que reconhecem essas relações e permitem a análise da sustentabilidade pela agregação de todos os índices.

Quando a relação é positiva:

$$
\mathrm{I}=(\mathrm{x}-\mathrm{m}) /(\mathrm{M}-\mathrm{m})
$$

Quando a relação é negativa:

$$
\mathrm{I}=(\mathrm{M}-\mathrm{x}) /(\mathrm{M}-\mathrm{m})
$$

Onde:

I = índice calculado para cada estado e municípios analisados;

$\mathrm{x}=$ valor de cada variável em cada estado ou município;

$\mathrm{m}=$ valor mínimo identificado nessas localidades;

$\mathrm{M}=$ valor máximo identificado nessas localidades.

As informações referentes aos estados brasileiros e municípios da Paraíba foram obtidas pela pesquisa de dados secundários disponibilizados nos principais institutos de pesquisas e órgãos governamentais, acessíveis nos sites do Atlas de Desenvolvimento Humano (IDH 2000), Caderno de Informações de Saúde (DATA SUS), Sistema Nacional de Indicadores Urbanos (SNIU), Instituto Brasileiro de Geografia e Estatística (IBGE), da Caixa Econômica Federal (CEF), Secretaria do Comércio Exterior (SECEX) do Ministério do Desenvolvimento, Indústria e Comércio Exterior, FINBRA - Finanças do Brasil da Secretaria do Tesouro Nacional, Agência Nacional de Telecomunicações (Anatel), Tribunal Regional Eleitoral (TRE - PB) e o Tribunal Superior Eleitoral (TSE) e o Instituto de Pesquisas Educacionais Anísio Teixeira (INEP).

Após a transformação das variáveis em índices, foi realizada a agregação desses índices por dimensão pela média aritmética, chegando-se ao IDSM social, IDSM demográfico, IDSM econômico, IDSM político-institucional, IDSM ambiental e IDSM cultural dos estados e dos municípios da Paraíba. O IDSM final foi calculado por meio da média aritmética dos IDSMs das dimensões.

Para a representação desses índices referentes a cada variável, foram utilizadas o IDSM das dimensões e do IDSM final, um conjunto de cores que corresponde aos níveis de sustentabilidade explicitados para cada localidade. A figura 2 abaixo mostra a classificação e representação dos índices (variação 0 e 1) conforme uma escala definida:

\begin{tabular}{|c|c|c|}
\hline ÍNDICE $(\mathbf{0}-\mathbf{1})$ & COLORAÇÃO & NÍVEL DE \\
SUSTENTABILIDADE
\end{tabular}

Quadro 2: Classificação e representação dos índices em níveis de sustentabilidade. 
Fonte: MARTINS e CÂNDIDO (2008).

Nessa classificação, os índices de sustentabilidade, com valores entre 0,0000 e 0,2500, revelam um nível crítico de sustentabilidade; os índices com valores entre 0,2501 e 0,5000 demonstram um nível de sustentabilidade em alerta; os índices com valores entre 0,5001 e 0,7500 indicam um nível de sustentabilidade aceitável; e por último, os índices com valores entre 0,7501 e 1,0000 revelam um nível ideal de sustentabilidade.

De acordo com o cálculo dos índices para os estados e municípios e, por conseguinte, do enquadramento nessa escala de representação exposta no Quadro 2, os mapas dos estados e dos municípios foram ilustrados, possibilitando uma visualização dos níveis de sustentabilidade. Essa representação foi realizada para todas as variáveis de forma isolada, para cada dimensão a partir da agregação das variáveis e para o IDSM final composto pela agregação das dimensões, tanto para os estados brasileiros quanto para os municípios da Paraíba (Martins e Cândido, 2008).

Todo esse trabalho resultou em um conjunto de informações sobre os níveis de sustentabilidade dessas localidades, podendo ser visualizadas pelos mapas (do Brasil e da Paraíba) que se apresentam de significativa importância para avaliação das políticas públicas já implementadas e, principalmente, como orientação para o processo de tomada de decisão dos gestores públicos na definição de políticas adequadas ao processo de desenvolvimento local sustentado.

\section{APLICAÇÃO DA METODOLOGIA}

A diversidade de aspectos que envolvem a sustentabilidade, leva a necessidade de abordagens, a partir de uma visão abrangente e ao mesmo tempo integrada de suas dimensões e indicadores. Foi neste sentido que a metodologia foi construída, cuja aplicação para sua validação tomou como objeto de estudo os estados brasileiros e, de forma específica, os municípios do Estado da Paraíba.

\subsection{Níveis de sustentabilidade dos estados brasileiros}

A partir do cálculo de cada índice para cada estado brasileiro, foi realizada uma agregação mediante uma média aritmética de todos os índices para a composição do IDSM por dimensão (social, demográfica, econômica, político-institucional, ambiental e cultural) para cada estado, classificados e representados posteriormente em níveis de sustentabilidade (ideal, aceitável, alerta, crítico e sem informação). Para condensação e compreensão dos resultados obtidos, foi calculado o percentual para cada nível de sustentabilidade em relação a cada dimensão para o conjunto de estados brasileiros. Assim, a investigação apresentou os seguintes resultados, conforme explicitados na Tabela 1:

Tabela 1: Representação percentual dos níveis de sustentabilidade por dimensão para os estados brasileiros.

\begin{tabular}{|l|c|c|c|c|c|c|}
\hline \multirow{2}{*}{$\begin{array}{l}\text { Representação } \\
\text { do nível de } \\
\text { sustentabilidade }\end{array}$} & \multicolumn{5}{|c|}{ Percentual de estados brasileiros por dimensões (\%) } \\
\cline { 2 - 7 } & Social & Demográfica & Econômica & $\begin{array}{c}\text { Político- } \\
\text { institucional }\end{array}$ & Ambiental & Cultural \\
\hline Ideal & 3,70 & 0,00 & 3,70 & 0,00 & 3,70 & 3,70 \\
\hline Aceitável & 48,15 & 33,33 & 33,33 & 3,70 & 55,56 & 7,41 \\
\hline Alerta & 48,15 & 66,67 & 48,16 & 85,19 & 40,74 & 14,81 \\
\hline Crítico & 0,00 & 0,00 & 14,81 & 11,11 & 0,00 & 74,08 \\
\hline Sem informação & 0,00 & 0,00 & 0,00 & 0,00 & 0,00 & 0,00 \\
\hline Total & 100 & 100 & 100 & 100 & 100 & 100 \\
\hline
\end{tabular}

Fonte: Dados da pesquisa (2008).

Quanto ao nível de sustentabilidade social, foi verificado que apenas 3,70\% dos estados apresentaram sustentabilidade ideal; 48,15\% aceitável; 48,15\% em alerta; e nenhum apresentou nível crítico. As Regiões Sul, Sudeste e Centro-Oeste apresentaram os melhores índices sociais, 
enquanto os estados com piores índices estão concentrados na Região Norte e Nordeste do país, evidenciando as desigualdades regionais e a necessidade premente de direcionar políticas públicas para as regiões que revelaram índices inferiores. A Paraíba representa uma situação de alerta, ocupando a $18^{\mathrm{a}}$ posição em relação aos demais estados.

Quanto ao nível de sustentabilidade demográfica, verificou-se que nenhum estado apresentou nível ideal; 33,33\% aceitável; 66,67\% situação de alerta e nenhum estado nível crítico. Numa perspectiva das regiões do país, foi verificado que a sustentabilidade demográfica apresentase melhor na Região Norte e parte do Centro-Oeste, enquanto a Região Sul, Sudeste e Nordeste apresenta resultados menos favoráveis. A Paraíba revelou uma situação de alerta, ocupando a $25^{\mathrm{a}}$ posição em relação aos demais estados.

Em relação ao nível de sustentabilidade econômica foram revelados os seguintes resultados: 3,70\% dos estados apresentaram nível ideal; 33,33\% dos estados situação aceitável; 48,16\% em alerta e 14,81\% nível crítico. Os resultados evidenciam as fragilidades interna da economia do país e das relações econômicas externas, bem como, as desigualdades em decorrência da concentração dos rendimentos, onde a Região Sul, Sudeste e parte da Centro-Oeste, apresentaram níveis de sustentabilidade mais satisfatórios em relação à Região Norte e Nordeste. A Paraíba apresentou um nível de sustentabilidade crítico, ocupando a $24^{\mathrm{a}}$ posição em relação aos demais estados.

O nível de sustentabilidade político-institucional revelou uma situação de alerta em quase todo o país, uma vez que nenhum estado apresentou nível ideal de sustentabilidade; apenas 3,70\% apresentaram situação aceitável; enquanto 85,19\% dos estados apontaram situação de alerta; e $11,11 \%$, nível crítico. Nesse contexto, prevaleceram os resultados desfavoráveis em quase todo o país, onde apenas o Estado de Minas Gerais revelou um nível de sustentabilidade políticoinstitucional aceitável. Essa realidade, caracterizada pela situação crítica e de alerta, evidencia as fragilidades das instituições, distribuição inadequadas dos investimentos, participação política da população e o acesso restrito aos serviços que são relevantes para o exercício da cidadania e para a qualidade de vida da população. A Paraíba revelou nível de alerta, ocupando a $17^{a}$ posição em relação aos demais estados brasileiros.

No que concerne ao nível de sustentabilidade ambiental, constatou-se que apenas $3,70 \%$ dos estados apresentaram nível ideal; 55,56\% aceitável; 40,74\% em alerta e nenhum estado apresentou nível crítico. De acordo com os resultados, foi possível verificar que os melhores índices foram identificados na Região Sul, Sudeste, parte do Centro-Oeste e Nordeste, enquanto a Região Norte evidenciou os piores índices de sustentabilidade ambiental. É importante ressaltar que, apesar de haver na Região Norte a maior reserva natural e a maior biodiversidade brasileira, não há em todos os estados infraestrutura adequada em relação ao controle e tratamento da água destinada à população, tais como o abastecimento de água, rede de esgotamento sanitário e coleta de lixo. A Paraíba revelou nível de sustentabilidade ambiental em alerta, ocupando a $19^{a}$ posição em relação aos demais estados.

O nível de sustentabilidade cultural revela que apenas 3,70\% dos estados apresentaram nível ideal de sustentabilidade; $7,41 \%$ aceitável; $14,81 \%$ em alerta, enquanto $74,08 \%$ dos estados revelaram nível crítico. Esses resultados mostram que não existe valorização da cultura no país e isso, constitui um aspecto que impede o desenvolvimento das potencialidades das regiões e das pessoas, tendo consequências sociais irreparáveis que se refletem em níveis significativos de desigualdades regionais, miséria, violência, entre outros aspectos. A Paraíba revelou um nível crítico e, assim, a $11^{\mathrm{a}}$ posição em relação aos estados brasileiros, evidencia que a maioria dos estados apresentam um nível de sustentabilidade cultural inferior ao da Paraíba.

A partir do cálculo do IDSM por dimensão para cada estado, conforme já explicitado, foi realizada uma nova agregação pela média aritmética desses índices por dimensão para a composição do IDSM final de cada estado. Para melhor compreensão desses resultados, foi calculado o percentual para cada nível de sustentabilidade (ideal, aceitável, alerta, crítico e sem 
informação) em relação ao IDSM final para o conjunto de estados brasileiros, conforme Tabela 2 a seguir:

Tabela 2: Representação percentual dos níveis de sustentabilidade final para os estados brasileiros.

\begin{tabular}{|l|c|}
\hline Representação do nível de sustentabilidade & Percentual de estados brasileiros (\%) \\
\hline Ideal & 25,00 \\
\hline Aceitável & 74,07 \\
\hline Alerta & 0,00 \\
\hline Crítico & 0,00 \\
\hline Sem informação & $\mathbf{1 0 0}$ \\
\hline Total & \\
\hline
\end{tabular}

Fonte: Dados da pesquisa (2008).

De acordo com os resultados, foi possível verificar que nenhum estado evidenciou nível ideal de sustentabilidade; apenas $25,93 \%$ apresentaram aceitável; enquanto 74,07\% indicaram nível de alerta. É importante ressaltar que nenhum estado revelou nível crítico, evidenciando que o Brasil tem perspectivas positivas de melhorar sua sustentabilidade pela adoção de políticas que reconheçam as diferenças regionais como forma de valorizar as potencialidades e riquezas locais, a partir da introdução das condições adequadas para o processo de desenvolvimento local. A Paraíba apresentou um nível de sustentabilidade em alerta e a $23^{a}$ posição em relação aos demais estados brasileiros.

O nível de sustentabilidade dos estados brasileiros pode ser visualizada no mapa a seguir (Figura 3) pela representatividade do IDSM final para todos os estados brasileiros.

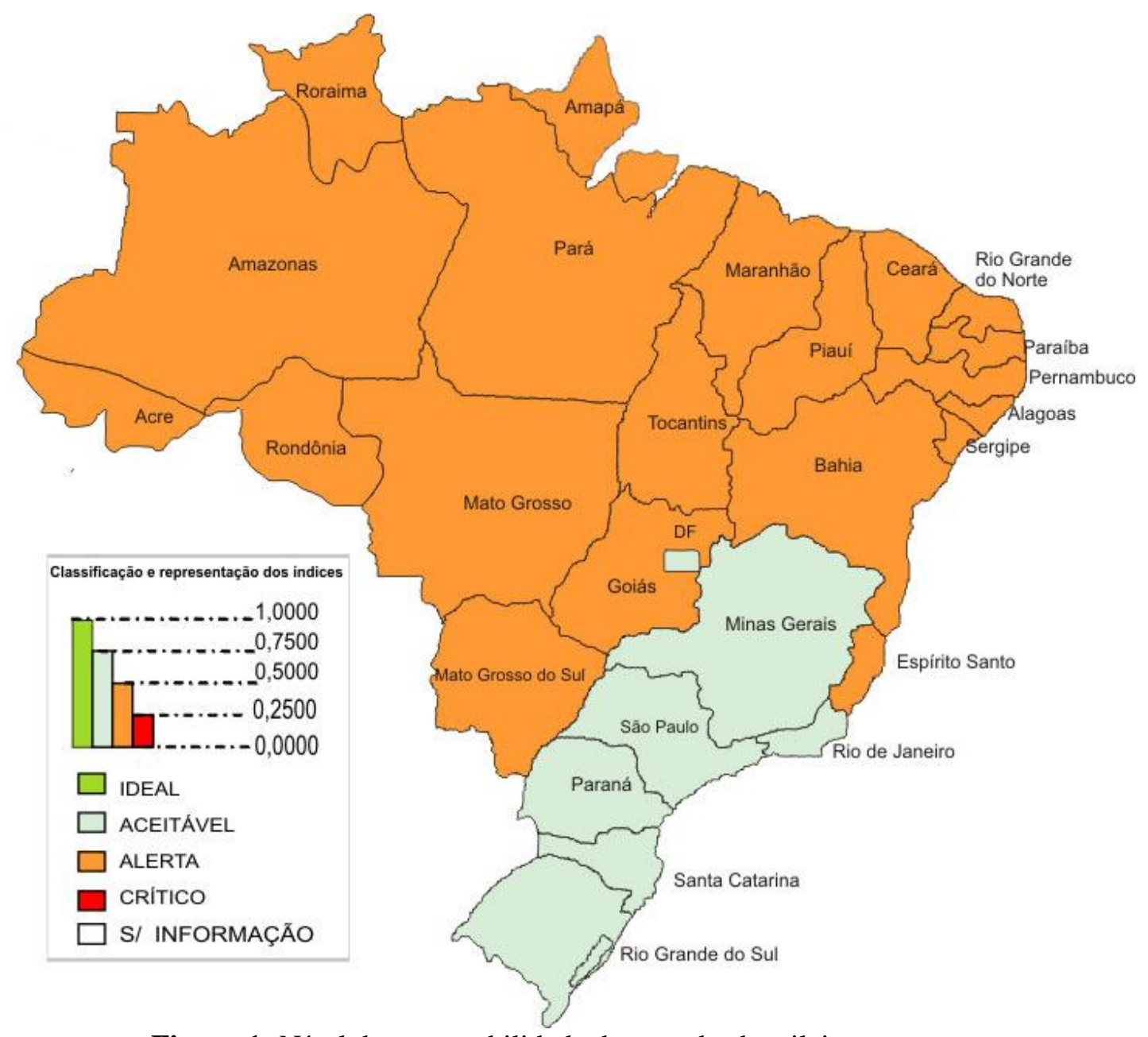

Figura 1: Nível de sustentabilidade dos estados brasileiros.

Fonte: MARTINS e CÂNDIDO (2008). 
Diante desses resultados, fica evidente que a Região Sul e Sudeste apresentaram níveis de sustentabilidade mais satisfatórios do que as Regiões Centro-Oeste, Norte e Nordeste, explicitando as desigualdades regionais existentes no Brasil. Nessa perspectiva, torna-se possível verificar a adequação das atuais políticas públicas, visando definir novas políticas de acordo com as necessidade e características evidenciadas por cada estado, no sentido de melhorar os níveis de sustentabilidade por meio de melhores resultados na sustentabilidade social, demográfica, ambiental, político-institucional, ambiental e cultural.

\subsection{Níveis de sustentabilidade nos municípios do estado da Paraíba}

A partir do cálculo de cada índice para cada município do Estado da Paraíba, foi realizada uma agregação mediante uma média aritmética de todos os índices para a composição do IDSM por dimensão (social, demográfica, econômica, político-institucional, ambiental e cultural) para cada município da Paraíba, classificados e representados posteriormente em níveis de sustentabilidade (ideal, aceitável, alerta, crítico e sem informação). Para compreensão dos resultados, foi calculado o percentual de cada nível de sustentabilidade em relação a cada dimensão para o conjunto de municípios da Paraíba. Esses resultados estão explicitados na Tabela 3:

Tabela 3: Representação percentual dos níveis de sustentabilidade por dimensão para o conjunto de municípios do Estado da Paraíba.

\begin{tabular}{|l|c|c|c|c|c|c|}
\hline \multirow{2}{*}{$\begin{array}{l}\text { Representação } \\
\text { do nível de } \\
\text { Sustentabilidade }\end{array}$} & \multicolumn{6}{|c|}{ Percentual de municípios da Paraíba por dimensões (\%) } \\
\cline { 2 - 7 } & Social & Demográfica & Econômica & $\begin{array}{c}\text { Político- } \\
\text { institucional }\end{array}$ & Ambiental & Cultural \\
\hline Ideal & 0,45 & 0,00 & 0,45 & 0,00 & 0,45 & 0,45 \\
\hline Aceitável & 32,74 & 39,46 & 2,24 & 0,45 & 57,85 & 0,45 \\
\hline Alerta & 66,81 & 60,54 & 97,31 & 77,58 & 41,70 & 0,45 \\
\hline Crítico & 0,00 & 0,00 & 0,00 & 21,97 & 0,00 & 98,65 \\
\hline Sem informação & 0,00 & 0,00 & 0,00 & 0,00 & 0,00 & 0,00 \\
\hline Total & 100 & 100 & 100 & 100 & 100 & 100 \\
\hline
\end{tabular}

Fonte: Dados da pesquisa (2008).

A análise do nível de sustentabilidade social nos municípios da Paraíba revelou que apenas 0,45\% dos municípios apresentaram um nível ideal de sustentabilidade; 32,74\% aceitável; 66,81\% em alerta e nenhum revelou nível crítico. Do ponto de vista do desenvolvimento sustentável, a Paraíba presencia um momento que requer algumas avaliações nos aspectos sociais, no sentido de buscar melhorias nas condições de habitação, saúde, educação e segurança e, assim, proporcionar mais qualidade de vida para a população.

A análise do nível de sustentabilidade demográfica nos municípios da Paraíba revelou que nenhum município apresentou nível ideal de sustentabilidade; 39,46\% nível aceitável; 60,54\% alerta; e nenhum nível crítico. De acordo com esses resultados, pode-se constatar que os municípios apresentam aspectos demográficos que não atendem aos objetivos da sustentabilidade, uma vez que, a grande maioria dos municípios apresenta baixa densidade demográfica, além de um contingente baixo de pessoas em idade ativa e produtiva.

Quanto ao nível de sustentabilidade econômica dos municípios da Paraíba constatou-se que apenas $0,45 \%$ dos municípios revelaram nível ideal; 2,24\% aceitável; enquanto 97,31\% uma situação de alerta e nenhum apresentou nível crítico. Diante dessa situação de alerta, torna-se necessário definir mecanismos para estimular o desenvolvimento da economia no Estado, por meio da elaboração de políticas e estratégias para identificação da vocação econômica dessas localidades, ampliação da capacidade de produção dos pequenos produtores, valorização das potencialidades locais e qualificação profissional, possibilitando a atuação adequada da população nas diversas atividades econômicas e, consequentemente, a geração de renda nesses municípios. Além disso, 
contribui para o fortalecimento das bases econômicas e a redução da dependência do município em relação aos recursos da União.

O nível de sustentabilidade político-institucional nos municípios da Paraíba revelou que nenhum município apresentou nível ideal de sustentabilidade; 0,45\% aceitável; enquanto 77,58\% revelaram situação de alerta e $21,97 \%$ nível crítico. Esse resultado revela as fragilidades do corpo institucional dos municípios e desperta para a necessidade de implementar políticas que estimulem a criação de instituições e serviços para a população.

Em relação ao nível de sustentabilidade ambiental dos municípios da Paraíba pôde-se constatar que apenas $0,45 \%$ dos municípios apresentaram nível ideal, 57,85\% aceitável, 41,70\% em alerta e nenhum município evidenciou nível crítico. A partir desses resultados, constata-se que as preocupações com as questões ambientais devem apresentar-se de forma mais efetiva nas decisões políticas, como forma de serem incorporadas de forma estratégica nas políticas públicas, para gerar resultados eficientes e eficazes para a população, proporcionando melhores condições de vida e mais qualidade ambiental.

O nível de sustentabilidade cultural apresentou resultados que evidenciam um quadro crítico, onde apenas $0,45 \%$ dos municípios apresentaram nível ideal; 0,45\% aceitável, $0,45 \%$ em alerta e a maioria absoluta, 98,65\% demonstraram nível crítico. Diante desses resultados em relação aos equipamentos culturais, pode-se afirmar que de acordo com os objetivos do desenvolvimento sustentável, essa realidade crítica revelada pelos índices evidencia uma necessidade urgente de políticas direcionadas para a cultura, que tenham como enfoque dotar os municípios com a infraestrutura e as condições adequadas de desenvolvimento local e sustentabilidade da região, pela criação de espaços para a prática de atividades culturais e artísticas.

Pelo cálculo dos IDSMs por dimensão para cada município do Estado da Paraíba, foi realizada uma nova agregação pela média aritmética desses índices por dimensão para a composição do IDSM final de cada município, representados em níveis de sustentabilidade. Foram calculados percentuais de cada nível de sustentabilidade (ideal, aceitável, alerta, crítico e sem informação) em relação ao IDSM final para o conjunto de municípios do Estado.

Tabela 4: Representação percentual dos níveis de sustentabilidade final para o conjunto de municípios.

\begin{tabular}{|l|c|}
\hline Representação do nível de sustentabilidade & Percentual de municípios da Paraíba (\%) \\
\hline Ideal & 0,00 \\
\hline Aceitável & 0,90 \\
\hline Alerta & 99,10 \\
\hline Crítico & 0,00 \\
\hline Sem informação & 0,00 \\
\hline Total Fonte: Dados da pesquisa (2008). & $\mathbf{1 0 0 \%}$ \\
\hline \multicolumn{2}{|c|}{} \\
\hline
\end{tabular}

De acordo com os resultados, nenhum município revelou nível ideal de sustentabilidade, 0,90\% nível aceitável, enquanto 99,10\% uma situação de alerta e nenhum evidenciou nível crítico, o que indica um quadro com perspectivas de melhoramentos.

Para melhor compreensão da sustentabilidade dos municípios da Paraíba, o mapa a seguir (Figura 2) mostra o nível de sustentabilidade identificado para todos os municípios. 


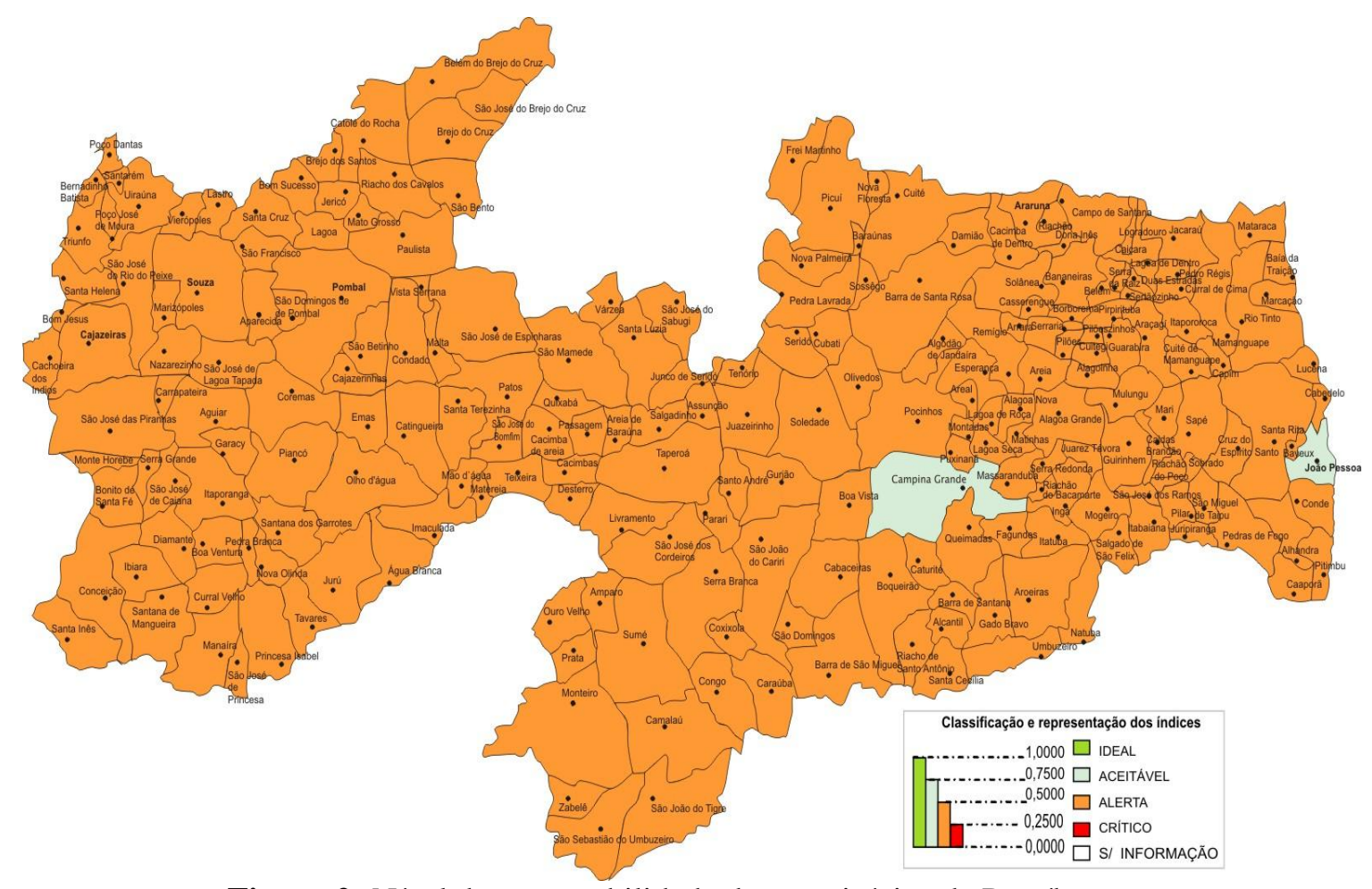

Figura 2: Nível de sustentabilidade dos municípios da Paraíba.

Fonte: MARTINS e CÂNDIDO (2008).

De acordo com o mapa acima (Figura 4), pode-se perceber a situação de alerta em que se encontra a sustentabilidade dos municípios da Paraíba, cujo resultado é compatível com a realidade da maioria dos estados brasileiros. Essa realidade requer avaliações para definir e redefinir políticas e estratégias adequadas que favoreçam o desenvolvimento sustentável.

\section{CONCLUSÕES}

Os resultados alcançados por este estudo configuram os objetivos iniciais delineados de propor uma metodologia para construção e análise do Índice de Desenvolvimento Sustentável para Municípios (IDSM), a partir da coleta, tratamento e análise de indicadores de sustentabilidade. A proposta metodológica com a transformação dos indicadores em índices de desenvolvimento sustentável pode contribuir para a viabilização do desenvolvimento sustentável, por meio de um retrato da realidade e a geração de informações que favorecem as interações entre os sistemas humanos e o ambiente para obtenção de objetivos sustentáveis.

Os resultados referentes a aplicação da metodologia para construir um índice de sustentabilidade possibilitaram uma investigação da realidade local, pela mensuração dos indicadores de sustentabilidade que forneceram informações para a identificação dos níveis de sustentabilidade social, demográfica, econômica, político-institucional, ambiental e cultural, e por conseguinte, o nível de sustentabilidade para os estados brasileiros e municípios da Paraíba. A classificação dos índices em níveis que representam a performance de sustentabilidade dessas localidades com posterior representação gráfica dos níveis de sustentabilidade em mapas (do Brasil e da Paraíba) permitiram melhor compreensão dos resultados e dos níveis de sustentabilidade revelada.

A partir da aplicação dessa metodologia foi possível verificar a sustentabilidade dessas localidades, e assim, retratar, de forma mais realista, os aspectos e as peculiaridades referentes as fragilidades e oportunidades, bem como, fatores que exercem maior influência na sustentabilidade dessas localidades. 
O conjunto de informações resultantes desse estudo permitiu questionamentos em relação a efetividade das políticas públicas implementadas, oferendo subsídios aos gestores públicos para a elaboração e adequação dessas políticas, com o intuito de vislumbrar melhorias nos aspectos sociais, demográficos, econômicos, políticos-institucionais, ambientais e culturais que evidenciaram situação crítica e de alerta, com vistas ao estabelecimento de um processo de desenvolvimento em bases sustentáveis e orientados para todo o contexto territorial considerado.

Diante dos resultados apresentados para os estados brasileiros e municípios da Paraíba, foi possível constatar a adequação da metodologia desenvolvida para a avaliação da sustentabilidade, que ofereceu significativas contribuições para o processo de decisão e implementação de políticas públicas e para o desenvolvimento local.

Essa proposta metodológica também mostrou-se de significativa relevância para estudos comparativos entre localidades, cujos resultados para o desenvolvimento sustentável consistem na possibilidade de redução das desigualdades regionais, pelo direcionamento de políticas adequadas às características locais, porém tendo como referência a realidade de todo o contexto ou território em análise.

Os resultados desse trabalho contribuem, de forma efetiva, para a ampliação do debate sobre o desenvolvimento sustentável em suas perspectivas teórica e aplicada, dando subsídios para que os gestores públicos se conduzam para os rumos adequados diante do processo de mudança para a construção de uma sociedade mais justa, a partir do desenvolvimento de forma equilibrada, equitativa e sustentável. Além dos resultados imediatos alcançados, esse estudo poderá ser replicado também em municípios de outros estados da Região Nordeste e demais regiões, ampliando sua relevância na avaliação das desigualdades regionais e locais, bem como, servindo de subsídio para orientação, redefinição e implementação de políticas voltadas para o desenvolvimento sustentável local.

Apesar do rigor metodológico da proposta torna-se crucial considerar algumas limitações, que se constituem em possibilidades para realização de novos estudos e pesquisas. A primeira, refere-se ao fato de que a escolha dos indicadores em cada uma das dimensões da sustentabilidade ocorreu a partir de sistemas de indicadores de sustentabilidade pré-existentes, o que leva a possibilidade de utilizar alguns indicadores que não tenham maior importância para o munício pesquisado. Além disso, nestes sistemas de indicadores de sustentabilidade todas as dimensões e indicadores de sustentabilidade têm o mesmo grau de importância, o que, na prática, não acontece, considerando que o contexto e as contingências específicas fazem com que existam graus de importância e prioridade diferentes. Para corrigir tais limitações, recomenda-se que nas próximas aplicações da metodologia, o processo seja mais participativo, onde os atores sociais e institucionais das localidades pesquisadas possam fazer a escolha dos indicadores e ponderá-los, utilizando técnicas de pesquisa como a análise fatorial e outras técnicas de análise multivariada.

\section{REFERÊNCIAS}

Agência Nacional de Telecomunicações - Anatel. Recuperado em outubro, 2008, de: http://sistemas.anatel.gov.br/sgmu/Localidade/Consolidado/frmListagem.asp

Atlas de Desenvolvimento Humano no Brasil - IDH 2000. Recuperado em janeiro, 2008, de: http://www.fjp.gov.br/produtos/cees/idh/atlas_idh.php.

Caderno de informações de saúde - DATA SUS. Recuperado em setembro, 2008, de: http://tabnet.datasus.gov.br/tabdata/cadernos

Caixa Econômica Federal - CEF. Recuperado em julho, 2008, de: https://www.beneficiossociais.caixa.gov.br/consulta/beneficio/04.01.00-00_00.asp. 
Comissão Mundial sobre Meio Ambiente e Desenvolvimento (1991). Nosso Futuro Comum. (2. ed.) Rio de Janeiro: Editora da Fundação Getúlio Vargas.

Estudos Amazônicos (NAEA). Universidade Federal do Pará, Belém. Resgatado em 15 de janeiro, 2008, de http://www.geocities.com/adagenor

Finanças do Brasil - Finbra. Recuperado em setembro, 2008, de: http://www.tesouro.fazenda.gov.br/estados_municipios/index.asp.

Franco, M.A.R. (2000). Planejamento ambiental para a cidade sustentável. São Paulo: Annablume: FAPESP.

Instituto Brasileiro de Geografia e Estatística- IBGE (2004). Indicadores de Desenvolvimento Sustentável: Brasil 2004. Diretoria de Geociências (Estudos e Pesquisas). Rio de Janeiro: IBGE.

Instituto Brasileiro de Geografia e Estatística-IBGE. Recuperado em setembro, 2008, de: http://www.ibge.gov.br.

Instituto Nacional de Estudos e Pesquisas Educacionais Anísio Teixeira- INEP. Recuperado em setembro, 2008, de: www.inep.gov.br.

Lafer, C. Abertura do seminário: O projeto CIEDS. (1996) In: Definindo uma agenda de pesquisa sobre desenvolvimento sustentável: Rio de Janeiro, 28-29 de novembro de 1994. Brasília: Fundação Alexandre Gusmão.

Martins, M. F., Cândido, G.A. (2008). Índice de Desenvolvimento Sustentável para Municípios (IDSM): metodologia para análise e cálculo do IDSM e classificação dos níveis de sustentabilidade - uma aplicação no Estado da Paraíba. João Pessoa: Sebrae.

National Research Council (1999). Mudanças e agressões ao meio ambiente: como a busca de melhoria e condições de vida dos homens tem contribuído para as mudanças ambientais em todo o mundo. São Paulo: MAKRON Books do Brasil.

Ribeiro, A. L. (2000). Sistemas de indicadores de sustentabilidade para a Amazônia. Tese (Doutorado em Desenvolvimento Socio-Ambiental). Programa de Pós-Graduação Interdisciplinar em Desenvolvimento Sustentável do Trópico Úmido, Núcleo de Altos.

Ruthes, S., Nascimento, D. E. (2006). Desenvolvimento sustentável e os arranjos produtivos locais. In: Simpósio de Administração da Produção, Logística e Operações Internacionais, 9. Anais... São Paulo: SIMPOI 1 CD ROM.

Schmidt, W., Cazella, A. A., Turnes, V. A. (1998). Indicadores de sucesso de processos de desenvolvimento local. Trabalho apresentado em "Oficinas sobre indicadores de sucesso em programas de desenvolvimento local”. Brasília/DF: PNUD.

Secretaria de Atenção a Saúde (CNESnet). Recuperado em setembro, 2008, de: $\underline{\text { http://cnes.datasus.gov.br/ }}$ 
Secretaria do Comércio Exterior - Secex. Recuperado em março, 2008, de: http://www.desenvolvimento.gov.br/sitio/sistema/balanca/.

Sepúlveda, S. (2005). Desenvolvimento microrregional sustentável: métodos para planejamento local. Brasília: IICA.

Sistema Nacional de Informações sobre Saneamento - SNIS. Recuperado em outubro, 2008, de: www.snis.gov.br

Sistema Nacional de Informações Urbanas - SNIU. Recuperado em julho, 2008, de:

www.sniu.gov.br

Tribunal Regional Eleitoral - TRE-PB. Recuperado em novembro, 2008, de: http://www.trepb.gov.br/resultados_eleicoes/online.htm

Tribunal Superior Eleitoral - TSE. Recuperado em novembro, 2008, de:http://www.tse.gov.br/internet/index.html.

Van Bellen, H. M. (2005). Indicadores de sustentabilidade: uma análise comparativa. Rio de Janeiro: Editora FGV.

Waquil, P.D., Shneider, S., Fileppi, E.E., Conterato, M.A., Specht, S. (2006). Avaliação de desenvolvimento territorial em quatro territórios rurais no Brasil. Recuperado em 05 de maio, 2010, de http://www6.ufrgs.br/pgdr/arquivos/508.pdf . Programa de Pós-Graduação em Desenvolvimento Rural (PGDR) da Universidade Federal do Rio Grande do Sul(UFRGS) - Porto Alegre.

Data do recebimento do artigo: 06/05/2010

Data do aceite de publicação: 30/03/2012 